\title{
WDM passive network design for small wavelength-count in local area/home applications
}

\author{
Kimio Oguchi · Tomoki Sakai · Dai Hanawa
}

Published online: 17 June 2011

(C) The Author(s) 2011. This article is published with open access at Springerlink.com

\begin{abstract}
Passive optical networks are attractive due to their enhanced reliability, reduced electrical power consumption, and small latency. This paper determines the feasibility of WDM (Wavelength Division Multiplexing) passive networks with low wavelength count for small environments such as buildings/homes from the viewpoint of the physical network configuration. After describing the general characteristics of WDM passive networks in the bus, star, and ring configurations, we elucidate the optical path losses to find design solutions when the three physical configurations are used to create small networks. It is concluded that the star configuration is the most feasible approach. The bus and ring are also feasible only for small areas and low wavelength counts.
\end{abstract}

Keywords Optical communication · WDM · Physical network configuration

\section{Introduction}

Fiber optic technologies have dramatically advanced since the actual commercial deployment of fiber optic systems

A part of this paper was presented at the 33rd International Conference on Telecommunications and Signal Processing (TSP2010) held in 17-20 August 2010 in Baden near Vienna, Austria.

K. Oguchi $(\varangle) \cdot$ T. Sakai · D. Hanawa Information Networking Laboratory, Graduate School of Science and Technology, Seikei University, 3-3-1 Kichijoji-Kitamachi, Musashino, Tokyo 180-8633, Japan

e-mail: oguchi@st.seikei.ac.jp

T. Sakai

e-mail:dm106209@cc.seikei.ac.jp

D. Hanawa

e-mail: hanawa@st.seikei.ac.jp in the early 80's. Core networks are transmitting, on a single fiber, several tens of wavelengths, each carrying a 10 or $40 \mathrm{~Gb} / \mathrm{s}$ channel. Total capacity is reaching the Terabit/second region. In access networks, the fiber-to-thehome (FTTH) system has spread rapidly in several countries. In Japan, more than 17 million users are already enjoying bandwidth-rich environments through FTTH [1]. Its major service is the Triple Play of broadband video (IP-TV), voice over Internet protocol (VoIP), and broadband Internet. Optical technologies have also penetrated into small areas: Local area networks (LANs) have been enhanced with the enhancement of Ethernet in terms of its bit rate and distance. They use optical modules such as SFP (Small Form factor Pluggable) modules in the short or long wavelength region for linking routers/bridges. Some home appliances (HA) use an optical digital link [2] for connecting AV terminals to the display for better QoS (Quality of Service) or longer distance.

Among the networks described above, regardless of size, passive optical technology is used only in the FTTH application. It is also called the PON (Passive Optical Network). ${ }^{1}$ It has a star configuration that sets a fiber splitter, or an optical power splitter, between the OLT (Optical Line Terminal) in the telephone office and the ONUs (Optical Network Unit) in home premises for reducing total cable length, or cable cost. The passive optical network has also been examined recently due to its good ability to reduce power consumption, or $\mathrm{CO}_{2}$ emission. However, it has the technical issue of how to configure the physical network, especially for point-to-multipoint (PtoM) or multipoint-to-multipoint (MtoM) connections.

${ }^{1}$ Please refer to papers appeared in PON or FTTH sessions in $O F C N$ FOEC, ECOC, OECC conferences. 


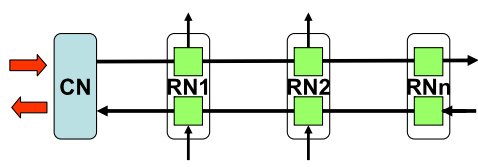

(a) Bus

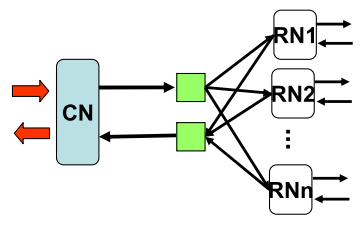

(b) Star

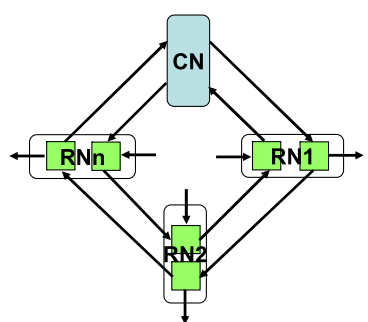

(c) Ring
Fig. 1 Network configurations considered: Bus, Star and Ring. CN denotes center node and RNi $i$ th-remote node

The purpose of this paper is to identify the most feasible physical configuration for WDM (Wavelength Division Multiplexing) passive networks when implemented in small areas such as buildings/homes. The configurations considered here are the bus, star, and ring.

Section 2 depicts the physical configurations considered and describes the general characteristics of WDM passive networks. Section 3 elucidates the optical path losses to find design solutions for the three physical configurations. Section 4 gives the conclusion and future work.

This paper is a modified version of the paper presented by the authors at the 33rd International Conference on Telecommunications and Signal Processing (TSP2010) [3].

\section{Network configuration and features}

\subsection{Network configuration}

Figure 1 depicts the high level functional blocks of the general three WDM configurations: (a) bus, (b) star, and (c) ring. We assume that the center node $(\mathrm{CN})$ and multiple remote nodes (RNs) are linked by 2 fibers. Each fiber is used on unidirectional WDM transmission basis. Bidirectional transmission lies outside the scope of this paper. WDM devices are set in each $\mathrm{RN}$ for bus and ring, and between $\mathrm{CN}$ and RNs for star. The $\mathrm{CN}$ transmits multiple wavelengths (e.g. $n$ wavelengths from $\lambda 1$ to $\lambda n$ ) downstream for bus and star, and both directions for ring. At each RN, dedicated wavelength(s) is (are) dropped and then optical-toelectrical $(\mathrm{O} / \mathrm{E})$ conversion is performed. The same wavelength(s) is (are) added after electrical-to-optical (E/O) conversion. Wavelengths other than the dedicated one(s) are passed through the $\mathrm{RN}$ for bus and ring.

\subsection{Characteristics of each configuration}

Generally speaking, passive networks offer higher network reliability and lower power consumption. The former is due to the use of passive optical devices rather than active ones. The latter is due to the absence of active devices such as $\mathrm{EO} / \mathrm{OE}$ converters, which consume electrical power in between the nodes.

Each configuration has several additional features as follows:

The bus is the simplest configuration as shown in Fig. 1(a) since several RNs are connected to each other in a cascaded manner. A WDM device at each RN simply drops a dedicated wavelength and passes the remaining wavelengths. The nearest $\mathrm{RN}$ to the $\mathrm{CN}$ may have highest priority in terms of traffic management because its propagation time, between the $\mathrm{CN}$ and $\mathrm{RN}$, is the shortest. The bus needs to have a mechanism that ensures network reliability. If some failure happens at any $\mathrm{RN}$ or link between nodes, the down stream side of the RN becomes isolated.

The star is the most popular passive optical network today and is used in the PON, or FTTH. A WDM device simply routes each dedicated wavelength to the corresponding output port. An AWG (Arrayed Waveguide Grating) is the best device to implement this function. The star can broadcast optical signals to multiple RNs simultaneously. If some failure happens at the WDM device or link between $\mathrm{CN}$ and the device, all RNs become isolated. If some failure happens on a link between the WDM device and a RN, only the corresponding $\mathrm{RN}$ becomes isolated.

The ring has a somewhat complicated mechanism as it has two rings that transmit in reverse direction. A WDM device at each RN simply drops a dedicated wavelength and passes the remaining wavelengths. This configuration is the most reliable. If some failure happens at any remote node or link between nodes, optical loop-back is performed which creates a protection ring at the cost of higher optical power consumption.

The deployment feasibility of each physical configuration relies strongly on the optical power budget of the network. The next section uses simplified network models to evaluate the optical path losses between the transmitter and receiver in order to clarify the application area of each network configuration.

\section{Path loss evaluations}

Given an optical power budget, network design is straightforward if the attributes of the optical entities in the network are mature. Since WDM systems for small area applications are still immature, design has to be performed with several estimated parameters. 
This section evaluates total path losses in terms of the optical power for the furthest link connection using estimated optical attributes for the bus, star, and ring configurations.

\subsection{Furthest link in each configuration}

In order to simulate the network, actual wiring routes are considered using a housing model [4] that consists of multiple stacked boxes, each with size of $a \times b \times c$ as shown in Fig. 2. Each building/house accommodates a NGW (Network Gateway) that connects to the optical outlet located on the wall of each room. Each terminal/home appliance is connected to the NGW through its nearest optical outlet, so each room has several optical outlets. It is also assumed that each room has a WDM device in the ceiling (left corner) if needed. Moreover, the ring type is structured as one ring per floor.

Previous evaluations $[5,6]$ indicate that the longest length for a typical Japanese house is less than $30 \mathrm{~m}$, and for most buildings less than $100 \mathrm{~m}$. For campus buildings the longest may not exceed $300 \mathrm{~m}$, e.g. $a=60 \mathrm{~m}, b=15 \mathrm{~m}, c=4 \mathrm{~m}$.

Therefore, the path loss evaluations in the next subsection use the fiber lengths of the following 3 options as; (i) $\leq 30 \mathrm{~m}$, (ii) $\leq 100 \mathrm{~m}$, and (iii) $\leq 300 \mathrm{~m}$ considering local area/home applications.

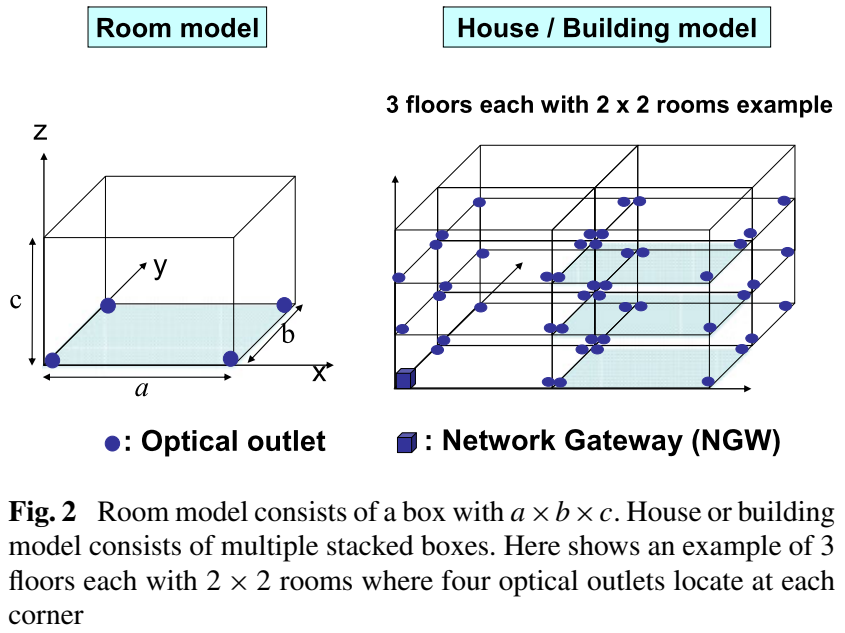

\subsection{Estimation of total path losses}

Path loss is here defined as the difference between the output at the CS and the input at the RN; it is the summation of the losses of all intervening optical entities [7]. Therefore, the loss (Lpath) is given as:

$L p a t h=L f i b+L b e n+L w d m+L c o n+M s y s$

where $L f i b$ : loss of fiber (product of per km loss and fiber length, lenf); Lben: fiber bending loss for installation in e.g. duct in building/home walls; $L w d m$ : loss of WDM device that is further categorized into Lmux for mux/demux loss, Lad for adding/dropping port loss in an ADM, Lthr for through port loss, Llop for loop-back loss in operation for protection; Lcon: connection loss for connector/splice; and Msys: system margin.

Table 1 summarizes several parameters for the bus configuration. Some parameters are common for all configurations. These values are provided by considering those of commercially available products, or estimation from recent research activities in this area:

- Number of wavelengths used $(\lambda): 4$ or 8 ,

- Fiber length (lenf): less than 30, less than 100 or less than $300 \mathrm{~m}$,

- Fiber loss (Lfib): 1.0, 5.0, 10.0 or $50.0 \mathrm{~dB} / \mathrm{km}$ considering both standard MM (Multi-Mode) -GOF (Glass Optical Fiber) and POF (Polymer Optical Fiber) in shorter wavelength region (e.g. $800 \mathrm{~nm}$ region CWDM [8]),

- Total bending loss (Lben): $2.0 \mathrm{~dB}$,

- Loss of WDM device for adding or dropping $(\operatorname{Lad})$ : 1.0, 2.0 or $3.0 \mathrm{~dB}$ for $4 \lambda \mathrm{s}$, and $2.0,4.0$ or $6.0 \mathrm{~dB}$ for $8 \lambda \mathrm{s}$,

- Loss of WDM for through port (Lthr): 2.0, 4.0 or $6.0 \mathrm{~dB}$ for $4 \lambda \mathrm{s}$, and 3.0, 5.0 or $7.0 \mathrm{~dB}$ for $8 \lambda \mathrm{s}$, and,

- Connection loss (Lcon): $0.5 \mathrm{~dB}$ per connector or splice with 10 pieces for $<30 \mathrm{~m}, 14$ for $<100 \mathrm{~m}$ and 18 for $<300 \mathrm{~m}$ for $4 \lambda \mathrm{s}$, and 18,22 or 26 for $8 \lambda \mathrm{s}$, and

- System margin (Msys): $2.0 \mathrm{~dB}$.

Table 1 Several parameters for the bus configuration

\begin{tabular}{|c|c|c|c|c|c|c|c|}
\hline \multirow[t]{2}{*}{ Wavelength count } & \multicolumn{6}{|l|}{ Items } & \multirow[t]{2}{*}{ Note } \\
\hline & 4 & & & 8 & & & \\
\hline lenf $[\mathrm{m}]$ & $\leq 30$ & $\leq 100$ & $\leq 300$ & $\leq 30$ & $\leq 100$ & $\leq 300$ & \\
\hline$L f i b[\mathrm{~dB}]$ & $\multicolumn{3}{|c|}{1.0 / 5.0 / 10.0 / 50.0} \times$ lenf & $\multicolumn{3}{|c|}{1.0 / 5.0 / 10.0 / 50.0} \times$ lenf & $\mathrm{dB} / \mathrm{km}$ (MM-GOF, POF considered) \\
\hline Lben $[\mathrm{dB}]$ & \multicolumn{3}{|c|}{2.0} & \multicolumn{3}{|c|}{2.0} & total loss \\
\hline $\operatorname{Lad}[\mathrm{dB}]$ & \multicolumn{3}{|c|}{$1.0 / 2.0 / 3.0$} & \multicolumn{3}{|c|}{$2.0 / 4.0 / 6.0$} & add/drop loss \\
\hline Lthr $[\mathrm{dB}]$ & \multicolumn{3}{|c|}{$2.0 / 4.0 / 6.0$} & \multicolumn{3}{|c|}{$3.0 / 5.0 / 7.0$} & through path loss \\
\hline Lcon $[\mathrm{dB}]$ & $0.5 \times 10$ & $0.5 \times 14$ & $0.5 \times 18$ & $0.5 \times 18$ & $0.5 \times 22$ & $0.5 \times 26$ & $0.5 \mathrm{~dB} /$ connector or splice \\
\hline Msys $[\mathrm{dB}]$ & & 2.0 & & & 2.0 & & \\
\hline
\end{tabular}


Table 2 Several parameters for the star configuration

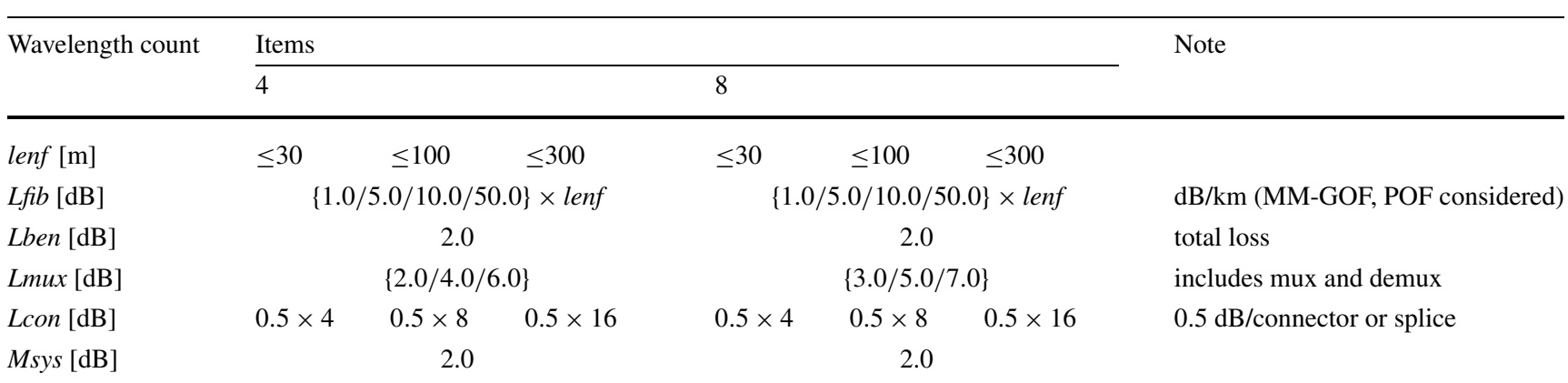

Table 3 Several parameters for the ring configuration

\begin{tabular}{|c|c|c|c|c|c|c|c|}
\hline \multirow[t]{2}{*}{ Wavelength count } & \multicolumn{6}{|l|}{ Items } & \multirow[t]{2}{*}{ Note } \\
\hline & 4 & & & 8 & & & \\
\hline $\operatorname{lenf}[\mathrm{m}]$ & $\leq 30$ & $\leq 100$ & $\leq 300$ & $\leq 30$ & $\leq 100$ & $\leq 300$ & \\
\hline$L f i b[\mathrm{~dB}]$ & $\multicolumn{3}{|c|}{1.0 / 5.0 / 10.0 / 50.0} \times$ lenf & $\multicolumn{3}{|c|}{1.0 / 5.0 / 10.0 / 50.0} \times \operatorname{lenf}$ & $\mathrm{dB} / \mathrm{km}$ (MM-GOF, POF considered) \\
\hline Lben $[\mathrm{dB}]$ & \multicolumn{3}{|c|}{2.0} & \multicolumn{3}{|c|}{2.0} & total loss \\
\hline $\operatorname{Lad}[\mathrm{dB}]$ & \multicolumn{3}{|c|}{$1.0 / 2.0 / 3.0$} & \multicolumn{3}{|c|}{$2.0 / 4.0 / 6.0$} & add/drop loss \\
\hline Lthr $[\mathrm{dB}]$ & \multicolumn{3}{|c|}{$2.0 / 4.0 / 6.0$} & \multicolumn{3}{|c|}{$3.0 / 5.0 / 7.0$} & through path loss \\
\hline Llop $[\mathrm{dB}]$ & \multicolumn{3}{|c|}{1.0} & \multicolumn{3}{|c|}{1.0} & loop-back loss \\
\hline Lcon $[\mathrm{dB}]$ & $0.5 \times 10$ & $0.5 \times 14$ & $0.5 \times 18$ & $0.5 \times 18$ & $0.5 \times 22$ & $0.5 \times 26$ & $0.5 \mathrm{~dB} /$ connector or splice \\
\hline Msys $[\mathrm{dB}]$ & & \multicolumn{3}{|c|}{2.0} & \\
\hline
\end{tabular}

Table 2 summarizes several parameters for the star configuration. They are almost the same as Table 1, but:

- Loss of the WDM device is that just for a simple mux/demux, and

- It has fewer connections than the bus.

Table 3 summarizes parameters for the ring configuration. They are almost the same as Tables 1 and 2, but:

- Loss of loop-back connection for network restoration is added, and

- Other values are similar to those for the bus in Table 1 as the network configuration is similar except the end of network is routed back to the $\mathrm{CN}$.

\subsection{Estimation results}

Figure 3 depicts the optical path loss for the bus with $4 \lambda \mathrm{s}$ calculated using the parameters in Table 1. As shown in the figure, WDM device loss dominates the path loss because the path includes several WDM devices e.g. 3 devices for a $4 \lambda \mathrm{s}$ ' bus as illustrated in the figure. If $L t h r$ is $6.0 \mathrm{~dB}$, the path loss could not illustrated in this figure as it has more than $30 \mathrm{~dB}$. Given the path loss of less than $20 \mathrm{~dB}$, the fiber loss of $<10.0 \mathrm{~dB} / \mathrm{km}$ is required with the WDM device losses of $2.0(\mathrm{Lthr})$ and $1.0(\mathrm{Lad}) \mathrm{dB}$, respectively, for lengths under $100 \mathrm{~m}$. For longer lenf, or higher fiber loss value, the bus could be difficult to realize as it uses so many connectors. Realizing a bus with $8 \lambda$ s could also be difficult as it has so many WDM devices.

Given the path loss of about $30 \mathrm{~dB}$, indicated for $\mathrm{Gb} / \mathrm{s}$ application [9], the combination of fiber length and fiber loss could be extended. For configuring the bus, however, the fiber loss of $50 \mathrm{~dB} / \mathrm{km}$ still presents some limit at longer lengths e.g. $>100 \mathrm{~m}$.

Figure 4 depicts the optical path loss for the star with $4 \lambda \mathrm{s}$ calculated using the parameters in Table 2. As shown in the figure, fiber loss dominates the path loss because the path includes only a pair of WDM mux and demux. Given the path loss of less than $20 \mathrm{~dB}$, the fiber loss of $<50.0 \mathrm{~dB} / \mathrm{km}$ is enough for any WDM mux/demux losses when lenf is less than $100 \mathrm{~m}$. This means that the current POF technology may apply for this configuration. For longer lenf, smaller Lmux and the fiber loss of $<10.0 \mathrm{~dB} / \mathrm{km}$ are needed. Although the optical path loss for the star with $8 \lambda \mathrm{s}$ is not depicted, the result is similar to that for $4 \lambda \mathrm{s}$.

Though optical path losses for the ring with 4 or $8 \lambda \mathrm{s}$ are not depicted, the results are similar to those for the 4 or $8 \lambda \mathrm{s}$ bus. One difference in terms of loss is the additional loss, $1.0 \mathrm{~dB}$, imposed by the optical loop-back operation, see Table 3 .

Figure 5 depicts the impact of the number of wavelengths/ RNs on optical path loss for (a) bus and (b) star 
Fig. 3 Optical path losses for 4-wavelengths bus configuration

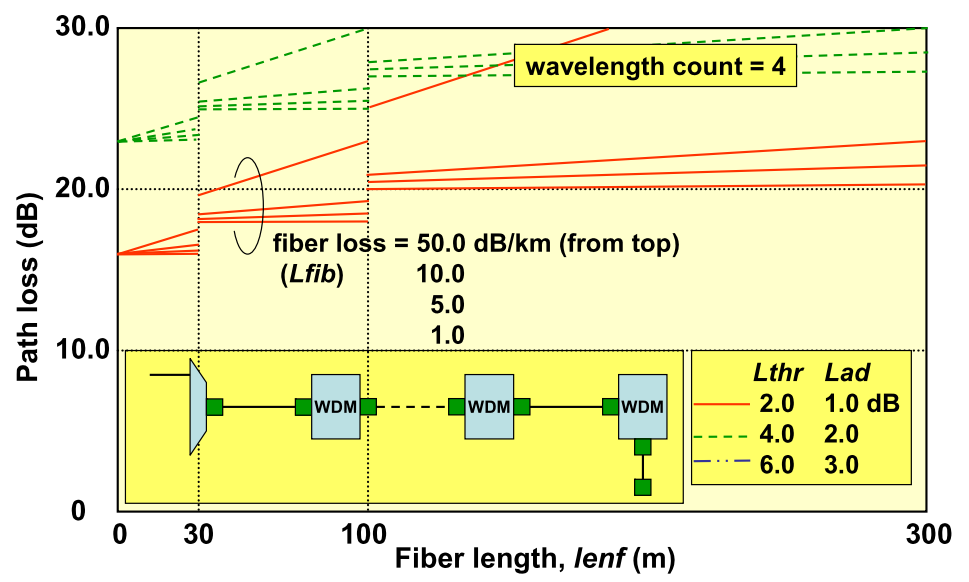

Fig. 4 Optical path losses for 4-wavelengths star configuration

Fig. 5 Optical path losses vs. the number of wavelengths/RNs for the bus and star configurations

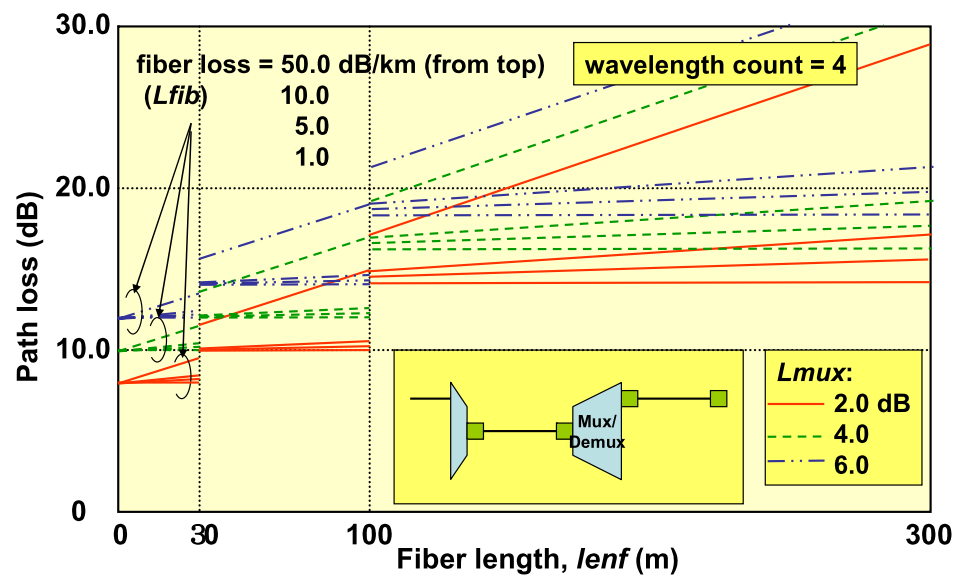

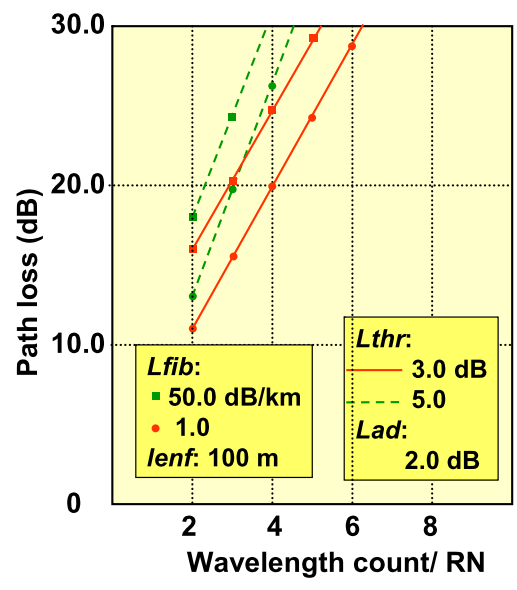

(a) Bus

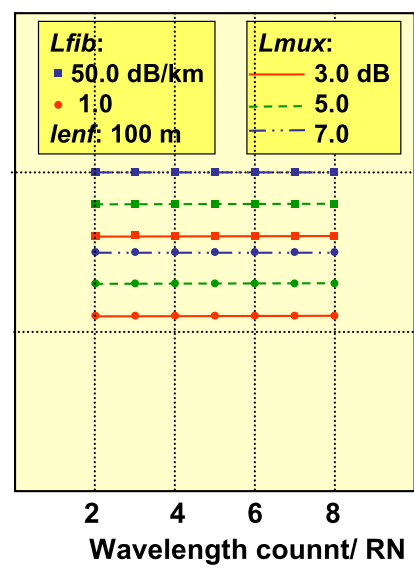

(b) Star configurations calculated using the parameters listed in Tables 1 and 2. Length of fiber, lenf is set $100 \mathrm{~m}$ with loss values of 50.0 or $1.0 \mathrm{~dB} / \mathrm{km}$. WDM device losses are $3.0 / 5.0 \mathrm{~dB}$ for the through port and $2.0 \mathrm{~dB}$ for the add/drop port in the bus; and 3.0/5.0/7.0 dB for the mux/demux loss for the star. The number of connectors in the bus depends on the number of the RNs connected. For example, if three RNs are connected, a total of seven connectors are needed, which cor- responds to $3.5 \mathrm{~dB}$ loss. As shown in the figure, the loss increases dramatically with the number of RNs in (a). As mentioned before, the bus sets several WDM devices between the CS and furthest RN yielding much higher path loss. On the other hand, the star has almost constant path loss regardless of the number of RNs as it has just one WDM device between the CS and any RN. Ring application in Fig. 5 is not shown, but it has almost the same performance as the 
Fig. 6 Optical path losses vs (a) loss of WDM and (b) connection loss

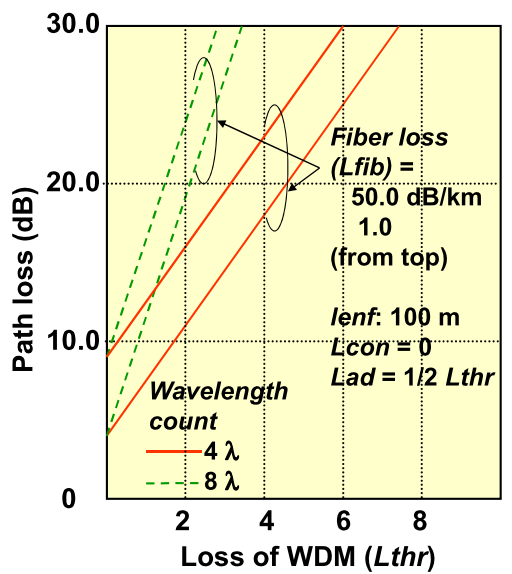

(a)

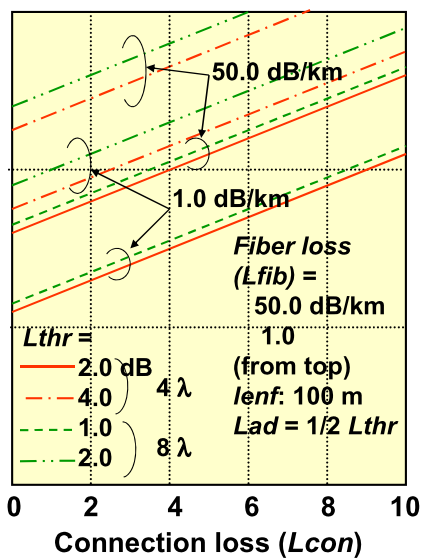

(b) bus except that the ring has an additional loss component due to loop back operation.

The simple estimation depicted in Fig. 5(a) clarifies that the bus has rather a limited applicable area in terms of several optical parameters. A further elucidation of WDM device loss and connection loss, dominant items in terms of path loss, is given below.

Figure 6 depicts the impact of (a) the loss of WDM devices and (b) connection loss on optical path loss for the bus configuration. As for the ring, the path loss is almost the same as that for bus as already described. Wavelength counts are 4 and 8 . Length of fiber, lenf is set to $100 \mathrm{~m}$ with loss values of 50.0 or $1.0 \mathrm{~dB} / \mathrm{km}$. Adding/dropping port loss $(L a d)$ is set to just half that for through port (Lthr). In Fig. 6(a), connection loss (Lcon) is set 0. In Fig. 6(b), Lthr is set 2.0/4.0 dB for $4 \lambda \mathrm{s}$ and $1.0 / 2.0 \mathrm{~dB}$ for $8 \lambda \mathrm{s}$. As seen in Fig. 6(a), lower WDM loss value is needed for the length of $100 \mathrm{~m}$ i.e. around $4 \mathrm{~dB}$ or less for $4 \lambda \mathrm{s}$ and $2 \mathrm{~dB}$ or less for 8 $\lambda$ s even with no connection loss given the path loss of $20 \mathrm{~dB}$. Even with the WDM loss value (Lthr) of 2.0/4.0 dB for $4 \lambda \mathrm{s}$ and $1.0 / 2.0 \mathrm{~dB}$ for $8 \lambda \mathrm{s}$, lower connection loss is also needed as depicted in Fig. 6(b) i.e. $10 \mathrm{~dB}$ or less for $2.0 \mathrm{~dB}$ for $4 \lambda \mathrm{s}$ and 1.0 for $8 \lambda \mathrm{s}$ with fiber loss of $1.0 \mathrm{~dB} / \mathrm{km}$ given the path loss of $20 \mathrm{~dB}$. Connection loss of $10 \mathrm{~dB}$ corresponds to 20 connectors/splices if each has $0.5 \mathrm{~dB}$ loss, so that the system can be easily configured even when several short pieces of fiber are connected or several devices are present in the system. However, for the fiber loss of $50 \mathrm{~dB} / \mathrm{km}$ or larger WDM loss (Lthr), only smaller connection values might be acceptable, i.e. a couple of $\mathrm{dB}$. This means that precise configuration design in terms of the number of connection and WDM devices will be needed for the system.

Even though an actual optical network design will demand more precise evaluations using the latest device values, the above results clarify that the star is feasible in all cases examined here, and that the bus and ring are also feasible when the WDM optical devices (e.g. WDM Add/Drop
Multiplexer) have low loss or the network is relatively small. It is also clarified that if the number of connections i.e. optical connectors or fiber splices, is reduced, network size can be enhanced.

\section{Conclusion and future work}

Passive optical networks are attractive due their good reliability, high electrical power efficiency, and small latency. This paper determined the feasibility of WDM (Wavelength Division Multiplexing) passive networks with low wavelength counts for small areas such as buildings/homes from the viewpoint of physical network configuration. After describing the general characteristics of WDM passive networks in the bus, star, and ring configurations, we elucidated the optical path losses to find design solutions when WDM devices are used in small networks based on the three physical configurations. It is concluded that the star configuration is most feasible, while the bus and ring are feasible only for small areas and low wavelength counts.

Following the network design described in this paper, the authors are prototyping passive WDM networks: the star with POF and the ring with the reconfigurable optical adddrop multiplexer (ROADM) node that uses dielectric thin film filters (TFs) for low wavelength count $(\leq 8)$ applications.

Acknowledgements The authors would thank Shohei Terada, Yu Kakishima and Shingo Yamakawa for discussions on the network model.

A part of this work was supported by MEXT. Grant-in-aid for Building Strategic Research Infrastructures.

Open Access This article is distributed under the terms of the Creative Commons Attribution Noncommercial License which permits any noncommercial use, distribution, and reproduction in any medium, provided the original author(s) and source are credited. 


\section{References}

1. Ministry of Internal Affairs and Communications, Japan (2010). Contract number for the broadband services. http://www. soumu.go.jp/ Accessed 6 July 2010.

2. Panasonic (2010). Optical Digital Cable. http://ctlg. panasonic.jp/product/info.do?pg=04\&hb=RP-CA20100. Accessed 15 Sept. 2010.

3. Oguchi, K., Sakai, T., \& Hanawa, D. (2010). In International conference on telecommunications and signal processing (TSP2010) (pp. 367-371).

4. Oguchi, K., Okodo, T., Tojo, K., \& Okada, K. (2006). In OFCNFOEC2006, JThB95.

5. Ministry of Internal Affairs and Communications (2003). Survey results of Japanese houses and lands. http://www.stat.go. jp/data/jyutaku/2003/index.htm. Accessed 26 March 2010.

6. Kakishima, Y., Terada, S., Hanawa, D., \& Oguchi, K. (2008). In ICCCS2008, A4-03.

7. Oguchi, K., Terada, S., Kakishima, Y., Yamakawa, S., \& Hanawa, D. (2009). POF2009, Paper 96.

8. E-LambdaNet (2010). HDMI-Optical Transmission. http://www. e-lambdanet.com/eng.html. Accessed 1 Oct. 2010.

9. Kim, K. H., Jo, S. C., Park, C. W., \& Park, S. H. (2010). In POF 2010 .

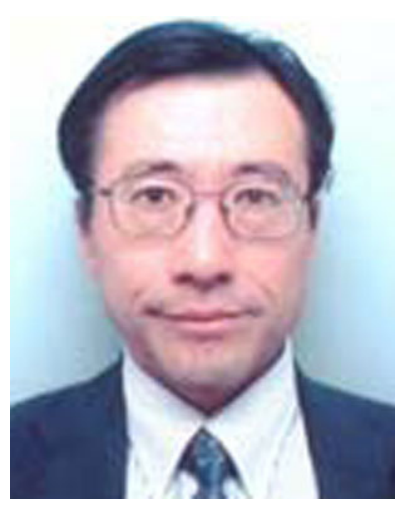

Kimio Oguchi received the B.E. and M.E. degrees in applied physics, and Dr. Eng. degree in electrical engineering in 1978, 1980 and 1995, respectively from Nagoya University, Nagoya, Japan. He joined the Yokosuka Electrical Communication Laboratory, NTT, Japan in 1980. During April, 1980 to March, 2004, he was engaged in the research and development of optical systems, in particular, fiber-optic local area networks, advanced fiberoptic digital systems, ATM link systems, photonic transport networks, applications of advanced photonic devices, and large contents distribution systems. He was involved in the standardization activities in both ITU-T SG's 13 and 15. He now is a Professor of the Information Networking Laboratory, Graduate School and Science and Technology and Faculty of Science and Technology, Seikei University, Tokyo, Japan. His research interests include information networking issues, applications utilizing e.g. sensor networks and optical networks, and vital functions, and contents distribution networks. He is recipient of the OECC'97 Best Paper Award, the NOC'97 Best Paper Award, the OECC'98 Best Paper Award, the Best Presentation Award at the 2001 and 2005 Annual Conference of Society for Running, and 2008 ICT Accomplishment Award of the ITU Association of Japan. He is a senior member of the IEICE, a member of IEEE Communications, IEEE Photonics, IEEE Computer, IEEE EMB Societies, OSA, ICST, Society for Running, Japanese Society for Emergency Medicine, Clinical Gait Analysis Forum of Japan, Digital Cinema Consortium of Japan, and CineGrid.

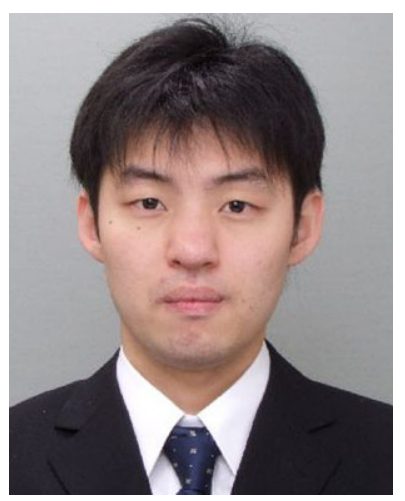

Tomoki Sakai is a Master student of the Graduate School of Science and Technology, Seikei University after receiving his bachelor's degree from Seikei University. His current interest is fiber-optic broadband networks and photonic routing network simulation tools. He is a student member of IEICE.

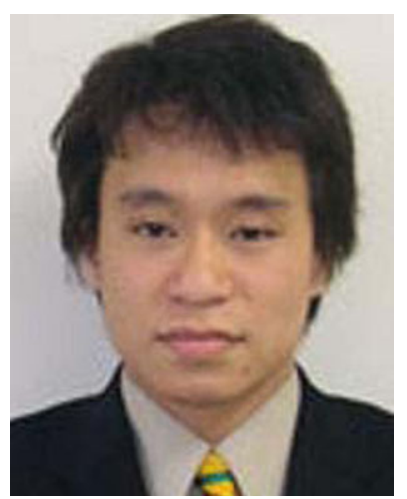

Dai Hanawa received the B.E., M.E., and Dr. Eng. degrees from Ibaraki University in 1999, 2001, and 2005, respectively. From 2005 to 2007, he was a Research Associate of the Department of Computer and Information Sciences at Tokyo University of Agriculture and Technology. Since 2007, he has been a Research Associate, and from 2008 he has been an Assistant Professor of the Department of Computer and Information Science at Seikei University. His research interests are in the area of human communications, especially the networked virtual environment, sensor network and its application. He received the first IEICE HCG Prize and IEICE MVE Prize 2009. He is a member of IEEE, ACM, IEICE, VRSJ and HIS. 